\title{
Monika Kulesza, L'amour de la morale, la morale de l'amour. Les romans de Catherine Bernard
}

\section{Franco Piva}

\section{(2) OpenEdition}

1 Journals

\section{Édition électronique}

URL : https://journals.openedition.org/studifrancesi/4671

DOI : 10.4000/studifrancesi.4671

ISSN : 2427-5856

Éditeur

Rosenberg \& Sellier

\section{Édition imprimée}

Date de publication : 1 avril 2012

Pagination : 142-143

ISSN : 0039-2944

\section{Référence électronique}

Franco Piva, " Monika Kulesza, L'amour de la morale, la morale de l'amour. Les romans de Catherine Bernard », Studi Francesi [En ligne], 166 (I | LVI) | 2012, mis en ligne le 30 novembre 2015, consulté le 19 novembre 2021. URL : http://journals.openedition.org/studifrancesi/4671 ; DOI : https://doi.org/ 10.4000/studifrancesi.4671

Ce document a été généré automatiquement le 19 novembre 2021.

\section{(c)}

Studi Francesi è distribuita con Licenza Creative Commons Attribuzione - Non commerciale - Non opere derivate 4.0 Internazionale. 


\title{
Monika Kulesza, L'amour de la morale, la morale de l'amour. Les romans de Catherine Bernard
}

\author{
Franco Piva
}

\section{RÉFÉRENCE}

MONIKA KULESZA, L'amour de la morale, la morale de l'amour. Les romans de Catherine Bernard, Warszawa, Unywerytet Warszawsky Wydzial Neofilolog, 2010, pp. 256.

1 Associer le nom d'un écrivain, comme Catherine Bernard, que la tradition littéraire a relégué, tout au plus, parmi les minores du Grand Siècle, et qui ne s'est illustré que dans le roman - genre futile et décrié s'il en fut - à celui de quelques-uns des plus grands moralistes de l'époque, tels La Rochefoucauld, La Bruyère et Fontenelle, peut paraître tenir du défi, ou de la provocation. C'est pourtant ce que Monika Kulesza a fait dans son travail, mais très sérieusement et sans la moindre intention provocatrice, à propos des romans de Catherine Bernard; non pas dans le but de bouleverser à tout prix l'échelle des valeurs littéraires du siècle de Louis XIV et le jugement que la critique a depuis longtemps porté sur les écrivains qui l'ont illustré, mais pour essayer de mettre finalement à la place qui leur est due un écrivain, Catherine Bernard justement, et une œuvre, ses romans essentiellement, que la critique paraît avoir, en effet, trop négligés ou, du moins, trop sous-évalués. Et celà grâce à une lecture attentive et lucide, libre de tout préjugé, de l'œuvre romanesque de Catherine Bernard et à une plus précise insertion de l'écrivain et de son œuvre dans le contexte idéologique et culturel à l'intérieur duquel l'un s'est formé et a opéré, et l'autre a pris corps.

2 Cette opération a mené Monika Kulesza à revoir, tout d'abord, la place et le rôle que dans la littérature des dernières décennies du XVII siècle a occupé le roman. Genre mineur, et fortement décrié par la critique contemporaine (essentiellement masculine), le roman fut pratiqué presque exclusivement par des femmes; il constitua en tout cas 
l'instrument privilégié par lequel elles purent porter sur la société dans laquelle elles vivaient un regard très lucide et souvent désenchanté. Car le roman, contrairement à ce qu'on croit d'habitude et à ce qu'ont dit dès l'époque ses dénigrateurs, ne fut pas un genre essentiellement léger, dont le but exclusif aurait été celui d'amuser ou de remplir plus ou moins agréablement, le temps des femmes ou des jeunes gens; il ne fut, du moins, pas que cela; et si les femmes ayant quelque chose à dire ont choisi ce genre, ce fut souvent parce que les autres (les genres soi-disant importants) leur étaient interdits. Or, ces femmes n'étaient pas non plus des esprits légers et superficiels: grâce aux salons qu'elles dirigeaient, ou qu'elles fréquentaient, elles participaient très activement à la vie intellectuelle et morale de l'époque, aux discussions qui y avaient lieu, aux problèmes, souvent très sérieux, qui y étaient débattus. Si elles ne s'occupaient que rarement de politique, elles affrontaient et se confrontaient à des problèmes tout aussi importants, tels que le rôle de la femme dans la société et dans ses rapports avec l'homme, la place que l'amour jouait, ou aurait dû jouer dans le couple, et dans l'aspiration de tout être humain au bonheur, le rapport que ce même bonheur avait avec l'au-delà, ou l'ici-bas, etc. Dans un contexte politique et idéologique de plus en plus fermé par l'absolutisme de Louis XIV, ces questions devenaient importantes, voire essentielles, et étaient traitées à la lumière des conditions dans lesquelles les hommes (et les femmes) étaient obligés de vivre: d'un côté, le salon, endroit clos dans lequel ils se formaient mais aussi se scrutaient, et étaient obligés, pour survivre, de pratiquer les règles de ce qu'on appelle la 'vie mondaine', faite de légereté et d'agrément mais aussi d'obligations et de contraintes; de l'autre le climat de plus en plus sombre qui avait remplacé les enthousiasmes (plus ou moins héroïques) de la première moitié du siècle, fortement imprégné de ce jansénisme (psychologique autant que religieux) qui était lui-aussi le fruit, par beaucoup d'aspects, de ce déchantentement qu'avait produit le revirement soudain que la France connut au début de la seconde moitié du siècle, et qui avait suscité la réflexion des esprits les plus lucides, ceux-là même qu'on appelle ou qu'on appellera les "moralistes", dont le pessimisme s'exprimait dans des formules icastiques destinées à former vite un patrimoine auquel tous puisaient, dans la mesure où elles exprimaient le sentiment commun: les femmes aussi bien que les hommes.

C'est justement la confrontation des romans de Catherine Bernard avec les oeuvres des "moralistes", et en particulier du plus célèbre d'entre eux, La Rochefoucauld, et de celui, Fontenelle, qui pour des raisons liées à la vie même de l'écrivain, fut très proche, peut-être même intime, de Catherine Bernard, qui permet à Monika Kulesza de faire voir combien la vision que Catherine Bernard a de la condition humaine est proche de celle des "moralistes" de l'époque; combien, au fond, elle vise le même but qu'eux. Cela apparaît très évident dans le titre qu'elle donne à la collection de "nouvelles" ou "petits romans" qu'elle commence à publier en 1687, année où parait la première d'entre elles, Eléonor d'Yvrée: "Les malheurs de l'amour», dont on peut facilement déceler les prodrômes dans les deux œuvres de jeunesse de Catherine Bernard, Frédéric de Sicile, un petit roman encore tout imprégné d'esprit précieux, et Le Commerce galant, une sorte de roman épistolaire, que Monika Kulesza analyse très longuement, et à plusieurs reprises, dans son livre, comme si elle avait été fascinée par cette œuvre, qui pose pas mal de problèmes par son statut même, mais qui, s'il est bien de Catherine Bernard, ouvre des perspectives fort intéressantes, que les œuvres suivantes semblent confirmer et infléchir en même temps. 
4 Amuser certes, mais aussi, on dirait même avant tout instruire ses lecteurs: tel est le but que se propose Catherine Bernard; avec une conscience très nette du rôle qu'est réservé aux écrivains (hommes ou femmes peu importe) dont on trouve la trace évidente dans les "Avertissements" que Catherine Bernard met en avant de ses "nouvelles" et par lesquels elle semble vouloir aider ses lecteurs à bien saisir la signification profonde de ses oeuvres et leur portée "morale". «L'amour de la morale», son goût de l'observation lucide des hommes et de la société qui les entoure, et qui se résume souvent dans des "maximes" du même genre que celles qu'ont formulées, à la même époque, les "moralistes", a donc mené Catherine Bernard à définir «la morale de l'amour", une morale très amère et fort désenchantée, comme amère et désenchantée était la vision qu'elle avait du monde; un monde que l'écrivain ressent sans espoir, mais non pas sans perspectives. Fort critique à l'égard de la société et du monde dans lesquels les personnages de ses romans, tous voués au malheur, vivent leurs tristes aventures, Catherine Bernard est, malgré tout, ouverte sur l'avenir; un avenir qu'elle ne sait pas encore définir mais que l'histoire de la littérature et de la culture française nous disent être celui du XVIII ${ }^{\mathrm{e}}$ siècle. Catherine Bernard constitue en effet un chaînon important entre Madame de La Fayette et l'abbé Prévost.

5 Malgré la compétence et le sérieux avec lequel Monika Kulesza a reconstruit le contexte culturel et littéraire dans lequel les romans de Catherine Bernard s'insèrent de plein droit et la lucidité des analyses qu'elle a menées sur les différentes œuvres de l'écrivain, tous les problèmes n'ont pas été définitivement résolus: bien des questions restent en suspens (qui était véritablement Catherine Bernard? quels furent ses rapports avec le monde des lettres et, plus en général, avec la société de son époque? quels furent ses rapports avec Fontenelle? Le Commerce galant est-il un roman au sens propre du terme et doit-il être attribué exclusivement à Catherine Bernard, ou bien s'agit-il d'une correspondance à quatre mains, comme certains indices que l'auteur tend parfois à faire siens, laisseraient croire? etc.). L'ouvrage de Monika Kulesza, et la lecture des romans de Catherine Bernard qu'elle propose, marquent en tout cas une date importante pour la tentative, mise en place depuis quelque temps, de récupérer à la grande littérature un écrivain et une œuvre que la critique a trop négligés et, plus en général, pour donner aux femmes écrivains de la fin du XVII siècle (car Catherine Bernard n'est pas seule) le rôle qu'elles méritent indubitablement et par delà tout esprit sectaire. 